\title{
Is there a fecundity/longevity trade-off under heat stress?
}

\author{
Nataly Gruntenko \\ Department of Insect Genetics \\ Institute of Cytology and Genetics \\ SB RAS \\ Novosibirsk, Russia \\ ORCID 0000-0003-3272-1518 \\ nataly@bionet.nsc.ru \\ Natalya V. Adonyeva \\ Department of Insect Genetics \\ Institute of Cytology and Genetics \\ SB RAS \\ Novosibirsk, Russia \\ nadon@bionet.nsc.ru
}

\author{
Evgenia K. Karpova \\ Department of Insect Genetics \\ Institute of Cytology and Genetics \\ SB RAS \\ Novosibirsk, Russia \\ karpova@bionet.nsc.ru
}

Petr N. Menshanov

Laboratory of Functional Neurogenomics Institute of Cytology and Genetics SB RAS

Novosibirsk, Russia

eternity@bionet.nsc.ru

\author{
Elena V. Burdina \\ Department of Insect Genetics \\ Institute of Cytology and Genetics \\ SB RAS \\ Novosibirsk, Russia \\ bella79@list.ru
}

Inga Yu. Rauschenbach

Department of Insect Genetics

Institute of Cytology and Genetics SB RAS

Novosibirsk, Russia

iraushen@bionet.nsc.ru

\begin{abstract}
The idea of this study was to discover remote consequences of the long-term effects of regular stress exposure. We investigated the effect of short-term heat stress $\left(38^{\circ} \mathrm{C}, 1 \mathrm{~h}\right)$ of varying frequency on longevity, fecundity, fat content, dopamine metabolism and resistance to acute heat stress $\left(38^{\circ} \mathrm{C}, 4 \mathrm{~h}\right)$ in Drosophila melanogaster. We showed that stress once a day causes a significant decrease in both longevity and fecundity, as well as in the fat content, and increases dopamine metabolism and survival under acute stress. We believe that this decrease could possibly contribute to adaptation, allowing insects to save energy. We found that weekly stress in the first two weeks does not affect the longevity, dopamine metabolism and resistance to acute stress, but causes a significant increase in the total level of fertility, despite sharp fertility drops on the exact days of stressing. Thus, two stress modes were found to have opposite effects on the reproductive function of $D$. melanogaster (negative or positive). However, the data obtained allow us to assume that there is no fecundity/longevity trade-off under heat stress. The trade-off we can see here is between reproduction and stress resistance. The flies use their energy resources either on the immediate individual survival (under acute stress) to the detriment of fertility and longevity or on the fecundity increase (under mild stress).
\end{abstract}

Keywords - Drosophila melanogaster, Longevity, Fecundity, Heat stress, Dopamine, Stress resistance

\section{Introduction}

The ability to develop an adequate response to unfavourable conditions of different natures is of crucial importance for the successful adaptation of an organism to ecological challenges. It is known that stress can cause both a decrease and an increase (due to the hormetic effect) in fitness [1]. Many genetic mechanisms, both ensuring resistance to unfavourable factors and underlying the processes of reproduction and ageing are highly conservative, and therefore model objects such as Drosophila melanogaster (Diptera: Drosophilidae) can be used for their study.

\section{Results and discussion}

Here we studied the effect of repeated episodes of mild heat stress $\left(38^{\circ} \mathrm{C}, 1 \mathrm{~h}\right)$ of varying frequency (once a day, once a week, twice in the first two weeks) on the longevity, fecundity, fat content, dopamine metabolism and resistance to acute heat stress $\left(38^{\circ} \mathrm{C}, 4 \mathrm{~h}\right)$ in D. melanogaster. We found that mild heat stress repeated weekly in the first two weeks did not affect the longevity, resistance to acute heat stress and dopamine metabolism, but caused a decrease in the fat content and increase in the total level of fertility, in spite of a sharp fertility drop on the exact days of stressing. Stress once a week throughout the entire period of reproduction did not affect the total level of fertility, although it resulted in a significant fertility decrease on the exact days of stressing. The mild stress repeated daily caused a significant longevity and fecundity decrease throughout the entire reproduction period. We believe that this decrease could possibly contribute to the adaptation, allowing flies to save energy. This suggestion is supported by the data on dopamine metabolism and fat content in the flies exposed to the mild stress once a day. Dopamine is known to be involved in the control of many cellular and physiological processes in insects, the neuroendocrine stress response and energy metabolism among them [2-4]. The fat body is the most important energy store of insect organism and a key organ in the metabolism of lipids and carbohydrates [5]. We found out that the mild heat stress repeated daily within two weeks resulted in an increased activity of the dopamine metabolism enzymes, dopamine-dependent arylalkylamine $\mathrm{N}$-acetyltransferase and alkaline phosphatase, which, together with an increased survival rate of these flies under acute heat stress, indicates a decrease in dopamine levels [6-8]. These data agree well with the results of transcriptome analysis of the fat body, which demonstrated significant changes in expression levels of genes involved in catecholamine and carbohydrate metabolic processes following the daily mild heat stress. The revealed decrease in the fat content following the mild heat stress repeated daily also supported the idea of energy consumption of adaptation process.

\section{Conclusions}

Thus, the data obtained allow us to assume that there is no fecundity/longevity trade-off under heat stress. The weekly stress episodes during first two weeks have a hormetic effect on reproduction but do not decrease longevity, whereas the daily stress episodes result in a decrease in both fecundity and longevity. The trade-off we can see here is between long- and short-term strategies of adaptation. The flies may compromise the reproduction and stress resistance. They could use their energy resources either on immediate individual survival (under more severe stress) to the detriment of fertility and longevity or on the fecundity increase (under less severe stress). 


\section{ACKNOWLEDGMENT}

This research was supported by the Russian Foundation for Basic Research (Project \# 19-04-00458).

\section{REFERENCES}

[1] É. Le Bourg, "Using Drosophila melanogaster to study the positive effects of mild stress on aging", Experimental Gerontology, 2011, V. 46, P. $345-348$.

[2] C. A. Martin, D. E. Krantz, "Drosophila melanogaster as a genetic model system to study neurotransmitter transporters", Neurochem Int., 2014, V. 73, P. 71-88.

[3] T. Ueno, J. Tomita, S. Kume, K. Kume, "Dopamine modulates metabolic rate and temperature sensitivity in Drosophila melanogaster", PLoS ONE, 2012, V. 7, e31513.

[4] M. E. Hanna, A. Bednářová, K. Rakshit, A. Chaudhuri, J. M. O’Donnell, N. Krishnan, "Perturbations in dopamine synthesis lead to discrete physiological effects and impact oxidative stress response in Drosophila”. J Insect Physiol., 2015, V. 73, P. 11-19.

[5] Z. Liu, X. Huang, "Lipid metabolism in Drosophila: development and disease", Acta Biochim Biophys Sin (Shanghai), 2013, V. 45, P. 44-50.

[6] E. V. Bogomolova, I. Yu. Rauschenbach, N. V. Adonyeva, A. A. Alekseev, N. V. Faddeeva, N. E. Gruntenko, "Dopamine down-regulates activity of alkaline phosphatase in Drosophila: the role of D2-Like receptors", J Insect Physiol., 2010, V. 56, P. 1155-1159.

[7] I. Yu. Rauschenbach, E. K. Karpova, N. V. Adonyeva, O. V. Andreenkova, N. V. Faddeeva, A. A. Alekseev, P. N. Menshanov, N. E Gruntenko, "Disruption of insulin signalling affects the neuroendocrine stress reaction in Drosophila females", JEB, 2014, V. 217, P. 3733-3741.

[8] E. V. Burdina, N. V. Adonyeva, E. K. Karpova, I. Yu. Rauschenbach, P. N. Menshanov, N. E. Gruntenko, "The effect of a mild heat stress of different frequencies on the adaptability of Drosophila melanogaster females", Archives of Insect Biochemistry and Physiology, 2019, V. 102, e21619. 